\title{
How Shape Constancy Relates to Drawing Accuracy
}

\author{
Dale J. Cohen and Holly Earls Jones \\ University of North Carolina Wilmington
}

\begin{abstract}
There is increasing evidence that the major source of drawing errors lies in the initial perception of the to-be-drawn object. In four experiments, the authors explore the relation between an artist's susceptibility to perceptual transformations, as measured by a simple shape constancy task, and drawing accuracy. The data reveal a robust negative relation between errors on the shape constancy task and drawing accuracy in general, and specifically the accuracy of the rendering of spatial relations. The data further suggest that the perceptual processes that lead to errors on the shape constancy task occur during the initial encoding of the stimuli. The authors conclude that the shape constancy task likely measures one's ability to overcome constructive perception processes that transform the retinal image into a final percept, and that this ability is necessary for the accurate rendering of objects.
\end{abstract}

Keywords: shape constancy, drawing, spatial perception, encoding, art

In $415 \mathrm{AD}$, Wang Wei hypothesized that adults' drawing errors arise as a result of perceptual transformations. Wang Wei stated that young artists should be wary of their perceptions of objects because, "the form of the object must first fuse with the spirit, after which the mind transforms it in many ways" (Sze, 1956, p. 39). Wei is describing the phenomenon whereby one's perception of a stimulus differs from the image of the stimulus that is projected onto the retina (i.e., the retinal image). This difference between the retinal image of a stimulus and the perceptual experience of that stimulus has been studied extensively (e.g., in the study of Gestalt perception, see Kohler, 1947; constructive perception, see Rock, 1983, 1996; among others). In the present paper, we briefly review the empirical evidence that perceptual transformations of the tobe-drawn stimulus is the major source of drawing errors and explore the relation between a simple shape constancy task and drawing accuracy. ${ }^{1}$

\section{Perceptual Transformations as the Major Source of Drawing Errors}

In an effort to identify the major cause of drawing errors, Cohen and Bennett (1997) decomposed the drawing process into four stages: accurately perceiving the to-be-drawn stimulus; deciding how and where to make marks to accurately represent the to-bedrawn stimulus; having the motor ability to make the mark; and evaluating the accuracy of one's drawing in an effort to identify and correct mistakes. Cohen and Bennett tested and ruled out the three latter stages (i.e., decision making, motor ability, and selfevaluation) as major causes of drawing errors. The authors concluded, by the process of elimination, that the first stage of the

Dale J. Cohen and Holly Earls Jones, Department of Psychology, University of North Carolina Wilmington.

Correspondence concerning this article should be addressed to Dale J. Cohen, Department of Psychology, University of North Carolina Wilmington, 601 South College Road, Wilmington, NC 28403-5612. E-mail: cohend@uncw.edu drawing process (perception of the to-be-drawn stimulus) is the most likely source of drawing errors.

The influence of perceptual transformation on the drawing process is relatively well documented (Blakemore, 1973; Blakemore, Carpenter, \& Georgeson, 1970; Deregowski, 1973; Freeman, 1980, 1987; Gregory, 1990; Lee, 1989; Reith, 1988; Van Sommers, 1984; Willats, 1997). Much of the evidence that perceptual transformations reduce drawing accuracy comes from experiments that study the effects of misperception resulting from shape constancy. Shape constancy refers to the phenomenon that obliquely presented shapes are perceived as less skewed than the shape projected on one's retina. So, for example, a rectangular door seen from an oblique angle will be perceived as more rectangular than the projection on the viewer's retina. A common explanation of this phenomenon is that the perceived orientation of the object influences the perceived shape of the object (e.g., Epstein \& Park, 1963; Epstein, Hatfield, \& Muise, 1977).

Mitchell, Ropar, Ackroyd, and Rajendran (2005) demonstrate a causal link between misperception of the to-be-drawn stimulus resulting from shape constancy and drawing errors. To test the effects of perception on drawing accuracy, the researchers asked adult participants to copy four parallelogram figures. Two of the figures were identical in size and shape, but shown in different orientations causing a misperception. The other two figures were identical to the first two in size, shape, and orientation, but perspective cues (i.e., table legs) were added causing the figures to appear as tables instead of simple parallelograms. The results showed that the drawings of the "tables" were less accurate than the drawing of the parallelograms. Mitchell et al. (2005) concluded that (1) the addition of the table legs induced participants to perceive the parallelogram as a table top that receded in space; (2)

\footnotetext{
${ }^{1}$ Here we use Cohen and Bennett's (1997) operational definition of a visually accurate rendering: "one that can be recognized as a particular object at a particular time and in a particular space, rendered with little addition of visual detail that cannot be seen in the object represented or with little deletion of visual detail" (p. 609, see Cohen \& Bennett, 1997 for an expanded definition).
} 
the effects of shape constancy led participants to misperceive depth cues; and (3) the misperception of depth cues caused the drawing errors. Shape constancy has also been found to influence the drawing accuracy of children (Lee, 1989).

If perceptual transformation is a major source of drawing errors, then it is clear that its influence can be overcome. That is, some people are able to draw realistic representations of stimuli and these people should be demonstrably less influenced by the perceptual transformations than those who cannot. Indeed, there is evidence that accomplished artists have superior visual cognition skills. Thouless (1932) showed that trained artists are less susceptible to the biases induced by shape constancy than the average observer. In a more thorough exploration of the relation between artistic skill and visual cognition ability, Kozbelt (2001) reveals that perceptual accuracy and drawing accuracy have a strong positive correlation. Kozbelt (2001) assessed the role of perception on drawing abilities by comparing the performance of artists and nonartists on a variety of drawing and perception tasks. The perceptual tasks included identifying the participants of blurred and incomplete pictures, finding visually camouflaged objects, and completing a mental rotation task. The data revealed that artists out-perform nonartists on all perceptual and drawing tasks. Kozbelt conducted a regression that revealed that performance on the perception tasks and the drawing tasks were positively correlated.

Although shape constancy has been shown to influence drawing accuracy, the relation between one's ability to overcome the effects of shape constancy and drawing accuracy have not been explored in detail. In the present paper, we present a detailed exploration of the relation between shape constancy and drawing accuracy. Specifically, over the course of a decade, our laboratory has been conducting experiments to assess the source of drawing errors. During that time, we started asking participants to complete a simple shape constancy task in addition to drawing tasks. It is now apparent that the shape constancy findings are robust and can, in themselves, be informative about the drawing process. Here, we present the relevant shape constancy results of these experiments. ${ }^{2}$ In Experiment 1, we validate a simple paper and pencil shape constancy task. In Experiment 2, we quantify the relation between shape constancy and drawing errors. In Experiment 3, we explore the influence of memory on shape constancy. Finally, in Experiment 4, we explore the relation between perceptual errors resulting from shape constancy and 1) feature and spatial drawing accuracy, and 2) recognition of features and their spatial relations.

\section{Experiment 1}

Experiment 1 was designed to assess shape constancy. This experiment was intended to validate the current methodology and stimuli.

\section{Method}

\section{Participants}

Forty-eight students volunteered to participate. Each volunteer was individually approached on the campus of a regional university in the southeast United States and asked to participate. Par- ticipants received no incentive for participation and were naïve with respect to the task.

\section{Stimuli}

Four photographs of an exterior window taken approximately $26^{\circ}, 52^{\circ}, 65^{\circ}$, and $78^{\circ}$ to the left of a frontal view of the window were used as stimuli (see Figure 1). The window was surrounded by a brick exterior. A response sheet was created that consisted of 21 polygons, four of which corresponded to the outlines of the four windows used as stimuli (see Figure 2). The remaining polygons were successive morphs between the outlines of the four windows used as stimuli. The polygons were arranged in order from most rectangular to least rectangular and assigned successive numbers from 1 to 21 . The four window outlines were the 6th, 12th, 15th, and 18th polygon, and the windows were termed according to their corresponding polygon (e.g., Window 6).

\section{Procedure}

Participants were approached individually and asked to participate in a brief experiment. Upon agreement participants were read the following instructions:

I am going to show you four photographs of the same window. Each picture will show the window at a different angle. I want you to look closely at the outline of the brick cutout around the window. After a brief delay, I will show you 21 different outlines. I want you to choose which of these outlines looks like the brick cutout of the window in the picture. Please try to disregard the fact that the picture represents an angled view of a rectangular window. Instead please pay very close attention to the view of the specific window in the photograph, and choose the outline on the answer sheet that matches that view perfectly.

Let me provide a quick example. Let us imagine a piece of cardboard that is cut into a square. Although this piece of cardboard is square, the shape of the cardboard cutout will vary with the angle that you view it. The cardboard cutout will appear square only when you view the cardboard from straight on. If you move to your right or left, the cardboard cut-out will appear as a trapezoid. I am interested in you matching the exact trapezoid that you see. Do you have any questions?

After indicating that they understood the directions, the participants were presented one of the four windows for $15 \mathrm{~s}$. Then the window was removed and the participants were immediately presented the response sheet. The participant then indicated, by pointing or stating the associated number, the outline that matched the window they were presented. The experimenter recorded the response. This process was repeated until the participant described all four windows. The order of presentation of the windows was counterbalanced between participants.

\section{Results}

Because the morphed polygons do not strictly correspond to natural projections of a rectangle from various angles (although no

\footnotetext{
${ }^{2}$ It should be noted that some of the included experiments were portions of a series of experiments exploring a drawing-related issue (e.g., the effects of practice on drawing). So as not to divert the reader's attention, we only present the shape constancy portions of the relevant experiments.
} 

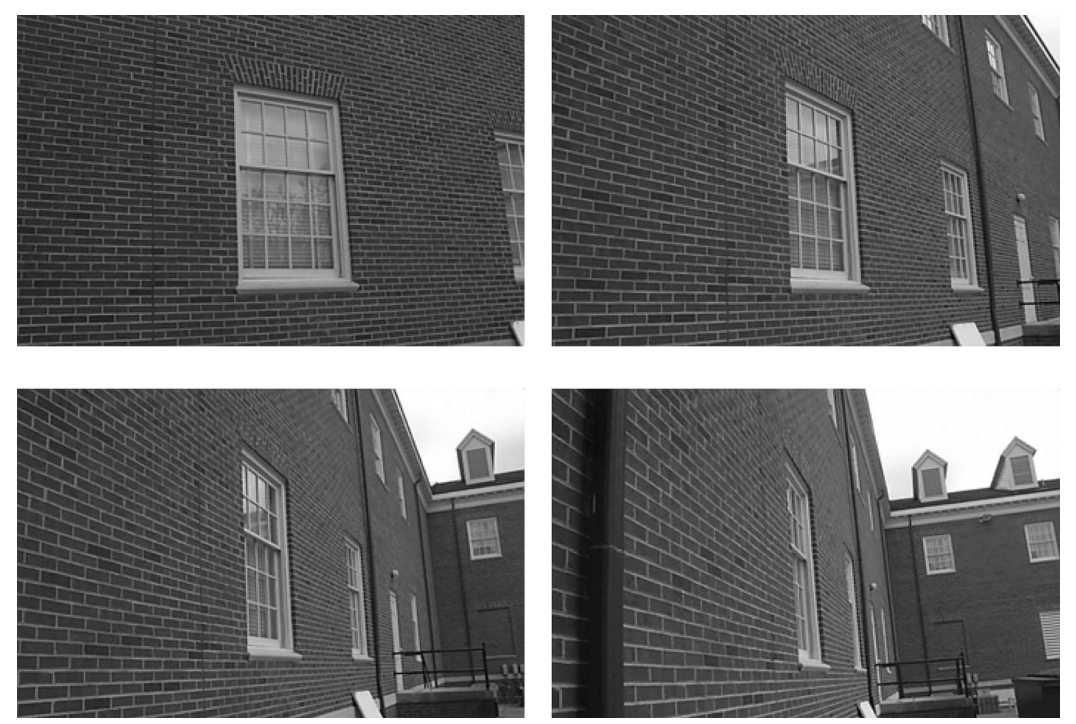

Figure 1. The stimuli in the shape constancy task.

participant was aware of this fact), we did not code the stimuli in terms of angles from upright. We therefore numbered each polygon in their ordinal relation to one another such that the rectangle was assigned the value "1" with increasingly nonrectangular polygons assigned increasing values (see Figure 2). As such, the correct responses for the four windows were $6,12,15$, and 18 , respectively. To assess accuracy, we calculated difference scores, where we subtracted the value assigned to the polygon chosen by

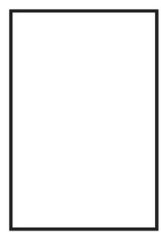

1

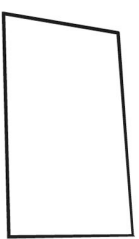

6

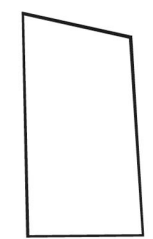

7

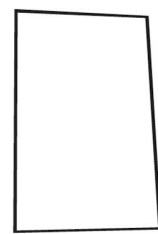

3

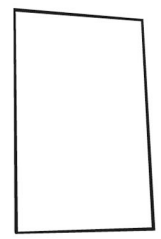

4

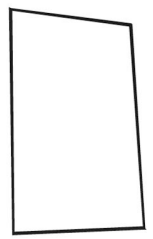

5

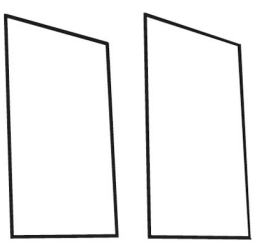

8

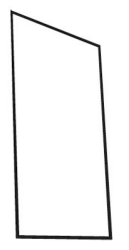

10

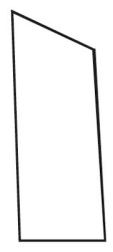

11

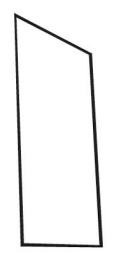

12

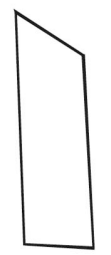

13

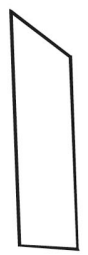

14
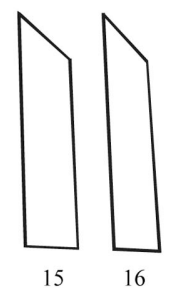

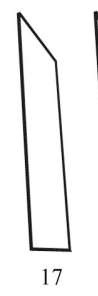

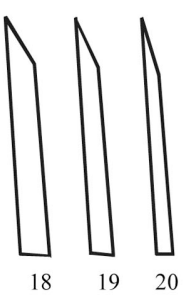

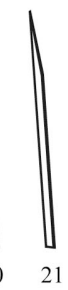

Figure 2. The response options for the shape constancy task. the participant from the actual polygon value for each window. Because the values were ordinally assigned, positive numbers indicated estimates closer to rectangle, while negative numbers indicated estimates farther from rectangular.

Table 1 contains the participants' average judgment error for each window view in Experiment 1 . We calculated a one-way (window angle) repeated measures ANOVA on the difference scores. The grand mean $(M=2.9, S D=3.1)$ was significantly greater than zero, $F(1,47)=132.3, p<.001, M S E=12.3, \eta^{2}=$ 2.81 ; indicating that participants estimated the shape of the window as closer to a rectangle (as predicted by shape constancy). There was also a significant effect of window, $F(3,141)=19.3$, $p<.001, M S E=6.84, \eta^{2}=0.41$. Tukey's HSD indicated that the participants' error when estimating the two extreme views $\left(26^{\circ}\right.$ and $78^{\circ}$ ) were equivalent to each other and significantly less than their error when estimating the two less extreme views $\left(52^{\circ}\right.$ and $65^{\circ}$ ), which were also equivalent to one another. Figure 3 presents a graph illustrating that the perceptual errors associated with the extreme views are less extreme than those associated with the more moderate views.

\section{Discussion}

The results of Experiment 1 replicate the findings of experiments demonstrating the effects of shape constancy (e.g., Thouless, 1931a, 1931b). Participants consistently chose an

Table 1

Means (SD) of Participants' Judgment Error for Each Window View in Experiments 1, 2, 3, and 4

\begin{tabular}{ccccc}
\hline View & Experiment 1 & Experiment 2 & Experiment 3 & Experiment 4 \\
\hline $26^{\circ}$ & $1.38(2.75)$ & $1.13(2.09)$ & $1.40(1.50)$ & $0.83(1.81)$ \\
$52^{\circ}$ & $3.81(2.77)$ & $4.41(3.02)$ & $3.22(2.41)$ & $3.56(2.63)$ \\
$65^{\circ}$ & $4.80(3.15)$ & $4.29(3.80)$ & $3.28(2.70)$ & $3.71(3.73)$ \\
$78^{\circ}$ & $1.67(2.75)$ & $2.43(3.79)$ & $1.08(2.13)$ & $1.65(2.73)$ \\
\hline
\end{tabular}




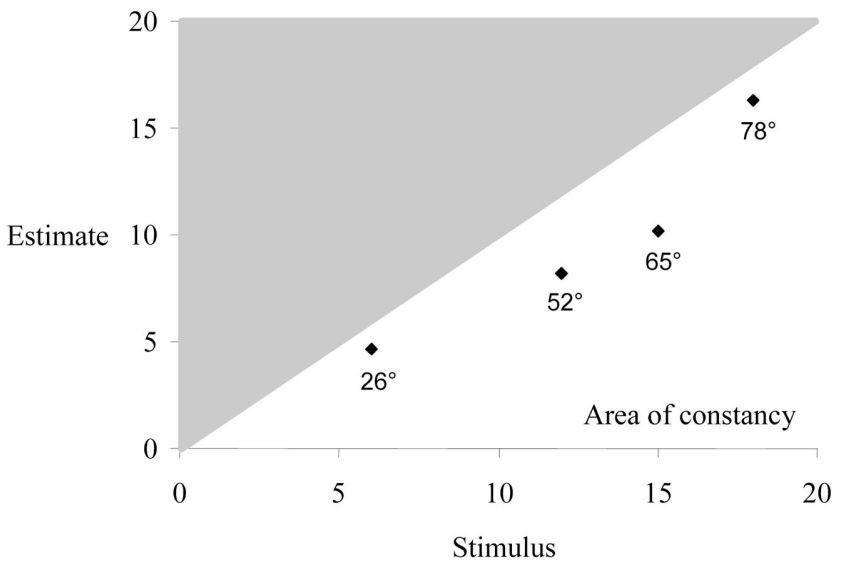

Figure 3. Plot of the participants' responses as a function of the stimulus presented in the shape constancy task.

outline representing a more "rectangular" view of the window than the view in the photographs. In Experiment 2, we assess the relation between accuracy in the shape constancy task and drawing accuracy.

\section{Experiment 2}

In Experiment 2, participants completed a drawing task in addition to the shape constancy task presented in Experiment 1. In the drawing task, participants were presented with a color photograph and asked to realistically render the image in the photograph. Participants were allowed $10 \mathrm{~min}$ to complete the drawing. The accuracies of these drawings were later judged by independent critics. Experiment 2 was part of a series of experiments exploring the relation between practice and drawing accuracy. Therefore, the participants were asked to draw the image several times in succession. Here, we only present the results relevant to shape constancy.

\section{Method}

\section{Participants}

Artists. Forty-eight participants from the general psychology subject pool volunteered to participate as artists. Here, the term artist refers to those participants who drew the stimulus. The term artist, as used here, implies no training or skill level. Only participants who reported no formal training in the visual arts were tested.

Critics. Fifty-one participants from the general psychology subject pool volunteered to participate as critics. Here, the term critic refers to those participants who rated the accuracy of the drawings. The critics had no formal training in the visual arts (please see Cohen, 2005, for rationale for using untrained critics). Participants received course credit for participation and were naïve with respect to the task.

\section{Materials}

We used two $6.75 \times 10.25$ in. $(17.5 \times 26.24 \mathrm{~cm})$ color photographs as stimuli for both the drawing and the critics' tasks. One photograph depicted the face and shoulders of an adult Caucasian woman looking directly into the camera (termed Photo A) and the other photograph depicted the face and shoulders of an adult Caucasian man looking directly into the camera (termed Photo B). The photographs were presented on a 15-in. VGA color monitor with a $60-\mathrm{Hz}$ refresh rate controlled by an 80486 microcomputer using the DOS operating system. The resolution of the monitor was $1,024 \times 768$

In the drawing task, artists used a pencil to draw on an $8.5 \times 11$ in. piece of white printer paper. The critics were provided the participants' original renderings. These stimuli were placed in transparent page protectors to increase durability and arranged in a three-ring binder.

\section{Procedure}

Drawing task. All artists were tested individually and were given a brief questionnaire concerning any formal training in drawing that they may have received, as well as their current drawing habits. The artists were then presented the same constancy task described in Experiment 1 with one exception: after the verbal instructions, the participants were presented an example. Participants were shown a picture of the window from straight on and then shown the window with an outline around the brick molding. This was done to make concrete the concept of the outline of the window. The drawing task followed the completion of the constancy task.

Each artist drew both photographs. Artists were asked to draw one photograph (e.g., Photo A) three times in succession, and then were asked to draw the other photograph (e.g., Photo B) once. Here, we only present the data from the first three renderings. The photographs were counterbalanced between participants. The instructions read to the artist were identical to those used by Cohen and Bennett (1997). In short, the artist was asked to draw the photograph as visually accurate as possible. Visual accuracy was explained as photo realism (given the limits of the medium). The artist was further instructed that only the visual accuracy of the rendering was important and that aesthetics, style, and creativity were not valued. Aesthetic value, style, and creativity were explained, through the use of verbal examples, as the creative abstractions similar to that of Picasso or Matisse. All artists indicated they understood the task.

Artists sat at a table with a computer screen place approximately 3 feet in front of them. An $8.5 \times 11$ in. plain white paper and a pencil were placed directly in front of the participant on the table. The presentation of the stimuli was controlled by a computer. The computer presented the appropriate photograph for $10 \mathrm{~min}$. The 10-min allotment was sufficient since most artists indicated completion at that time (see Cohen \& Bennett, 1997). At the end of the 10-min time period, the experimenter removed the participant's drawing and placed a new white sheet of paper in front of the participant. This procedure was repeated for each presentation of a photograph.

Critics' task. All critics participated individually in the same room (with the same set-up) that the artists drew the photographs. A computer monitor approximately 3 feet in front of the critic presented the photographs of the faces individually (i.e., Photo A $\&$ B). Between the monitor and the critic, was a binder containing the original renderings produced in the artist's task of one of the photographs (e.g., all the renderings of Photo A). There were two 
binders (one for each face), each containing 96 original renderings. The photograph of the face corresponding to the renderings was presented on the computer monitor. The critic was told to rate the rendering's visual accuracy on a scale from 1 to 20 , with 1 indicating a very poor representation and 20 indicating a very accurate representation. The critic was given the same explanation of visual accuracy as the participants. The critic was permitted to take as much time as he or she needed to assess each rendering. All of the critics indicated they understood the task.

When the critic completed his or her judgments of the renderings in the first binder, the critic was given the second binder containing the artists' renderings of the remaining photograph and the image on the computer monitor was changed to the photograph of the face corresponding to the renderings in the binder. The order of the binders was counterbalanced between critics. The order of the renderings in each binder was randomized between critics.

\section{Results}

To assess perceptual errors resulting from shape constancy, we calculated difference scores as in Experiment 1 (see Table 1). We calculated a one way (window angle) repeated measures ANOVA on the difference scores. The grand mean $(\mathrm{M}=3.06, S D=3.5)$ was significantly greater than zero, $F(1,47)=69.13, p<.001$, $M S E=26.1, \eta^{2}=1.47$; indicating that participants estimated the shape of the window as closer to a rectangle (as predicted by shape constancy). There was also a significant effect of stimulus, $F(3$, $141)=22.2, p<.001, M S E=5.4, \eta^{2}=0.34$. Tukey's HSD indicated that the participants' error when estimating the extreme $26^{\circ}$ view was significantly less than their error when estimating the two less extreme views $\left(52^{\circ}\right.$ and $65^{\circ}$ ) (see Table 1$)$, but the error associated with the extreme $78^{\circ}$ view was not significantly different from any other view. There were several large outliers in the direction predicted by shape constancy for the $78^{\circ}$ view window. The extra variance reduced the power of the analysis and resulted in the $78^{\circ}$ view condition not differing significantly with respect to the other conditions. As with Experiment 1, the error associated with the two less extreme views was equivalent.

\section{Reliability of Critics Ratings}

To ensure consistent use of the rating scale between critics, we standardized each critic's ratings to have a mean of zero and an $S D$ of one. These standardized ratings were used in all analyses.

Novice critics were selected, in part, because a large enough sample could be gathered so any individual critic would not have a large influence on the data. Nevertheless, it is important to assess the reliability of the critics' ratings. Cronbach's alpha, $\alpha=.97$, showed that, although novice critics were selected, the critics' ratings were remarkably reliable given the subjective nature of the task (a value greater than 0.7 is considered good reliability, Nunnaly, 1978).

The current dataset provides the information necessary to: (1) quantify the relation between perceptual errors resulting from shape constancy and drawing errors, and (2) quantify the relation between perceptual errors resulting from shape constancy and improvement due to practice. Perceptual errors resulting from shape constancy were calculated using the same method described in Experiment 1.

\section{Shape Constancy and Drawing Accuracy}

If reduced shape constancy relates to drawing accuracy, then there should be a negative relation between accuracy ratings and perceptual errors resulting from shape constancy. To assess this relation, we calculated a regression with average drawing accuracy (over all four drawings) as the criterion variable and the participant's judgment error for the Window 12 in the constancy task. We choose to use Window 12 because the largest constancy errors were elicited by Windows 12 and 15 and the constancy error for these two windows were not significantly different. We chose not to average all views (as we do in Experiment 4) because the extreme $78^{\circ}$ view in Experiment 2 showed a slightly different pattern than Experiments 1, 3, and $4 .^{3}$

The regression revealed that perceptual errors resulting from shape constancy were a significant predictor drawing accuracy, $F(1,46)=10.4, p=.002, r^{2}=.18$. Both the intercept $(0.4)$ and the slope $(-.09)$ were significant $(p<.05)$ (see Figure 4$)$. Adding a quadratic component did not significantly improve the fit (linear $r^{2}=.18$ vs. quadratic $r^{2}=.195$ ). The correlations between drawing accuracy of each of the four drawings and judgment error for Window 12 were all significant $(p<.05)$ and ranged from -0.35 to -0.43 . These data reveal that the relation between drawing accuracy and the effects of shape constancy is robust.

\section{Shape Constancy and Drawing Improvement}

A one-tailed paired $t$ test revealed that participants' third drawing was significantly more accurate than their first drawing, $t(47)=1.9, p=.03, d=0.27$. If reduced shape constancy relates to drawing improvement due to practice, then there should have been a negative relation between change in accuracy ratings between the first and third drawing (both of the same image) and perceptual errors resulting from shape constancy. To assess this relation, we calculated a regression with drawing improvement (rating of third drawing minus rating of first drawing) as the criterion variable and the participant's judgment error for Window 12 in the constancy task. The regression revealed that perceptual errors resulting from shape constancy was not a significant predictor drawing improvement, $F(1,46)=1.68, n s$.

\section{Discussion}

The data from Experiment 2 reveal a robust negative relation between drawing accuracy and errors in a shape constancy task. Indeed, perceptual errors resulting from shape constancy accounts for about $18 \%$ of the variance in drawing accuracy. There was no relation, however, between perceptual errors resulting from shape constancy and drawing improvement. This suggests that shape constancy is related to the ability to encode and/or store the image accurately rather then the ability to learn from and correct rendering errors.

The perceptual errors resulting from shape constancy recorded thus far may be a function of how the stimulus is encoded and/or how resistant one's memory is to distortion effects. In Experiment

\footnotetext{
${ }^{3}$ When analysis is run with the average of all window views, the same significant data pattern emerges. The correlation, however, is slightly weaker ( $r=.3$ vs. $r=.43$ ) due to some outliers in the extreme $78^{\circ}$ view.
} 


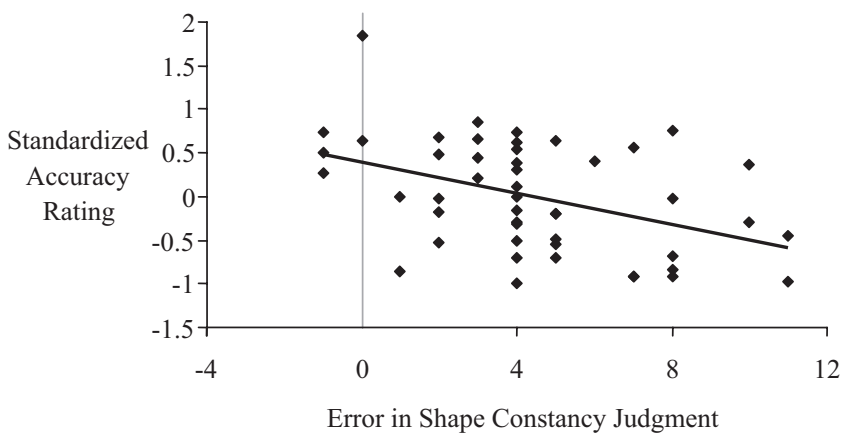

Figure 4. A plot of the relation between drawing accuracy and shape constancy.

3, we attempt to disambiguate the influence of encoding and storage on perceptual errors resulting from shape constancy. Disambiguating these two influences will help identify the relevant perceptual process in the shape constancy task that is related to drawing accuracy.

\section{Experiment 3}

In Experiment 3 we replicate the findings of Experiment 1 with the exception that we had five delay conditions: a simultaneous condition where the window and the response sheet were simultaneously visible, and four delay conditions. The simultaneous condition will provide a baseline of the perceptual effect of shape constancy because the stimulus will not need to be stored in memory before a response is made. All other conditions force participants to store the image in memory. If shape constancy is purely a perceptual phenomenon that is present at the time of encoding and there is no influence of memory distortion, then there should be no effect of delay. If there is an influence of memory distortion, participants' perceptual errors resulting from shape constancy should increase with delay time.

\section{Method}

\section{Participants}

One hundred and twenty students volunteered to participate. Sixty participants were male and 60 participants were female. Each volunteer was individually approached on campus of a region university in the southeast United States and asked to participate. Participants received no incentive for participation and were naïve with respect to the task.

\section{Stimuli}

The same four photographs that were used in Experiment 1 were also used in the present experiment. In addition, the same rating sheet as Experiment 1 was used in the present experiment, with the exception that the numbers were removed. The participants responded by pointing to the polygon that most resembled the outline of the window that they saw.

\section{Procedure}

There were three independent variables: Gender (male vs. female), Stimulus $\left(26^{\circ}, 52^{\circ}, 65^{\circ}\right.$, and $\left.78^{\circ}\right)$, and delay condition (simultaneous, immediate, 15-s, 60-s, and 150-s delay). The delay condition was included to assess the affect of memory in constancy. In the simultaneous condition, participants viewed the window and the rating sheet at the same time. The participants responded within a 15 -s time limit. In the immediate delay condition, the participants viewed the window for 15-s, and then the rating sheet immediately replaced the window. In the 15-, 60-, and 150-s delay conditions, the participant viewed the window for $15 \mathrm{~s}$, then the window was removed and there was either 15,60 , or $150 \mathrm{~s}$ before the rating sheet was presented. Stimulus was a withinsubjects variable, so all participants viewed every window. The order of presentation of the windows was counterbalanced between participants. Delay was a between-subjects variable and was counterbalanced between participants. Twelve males and 12 females participated in each delay condition.

Participants were approached individually and given the same instructions presented in Experiment 2. After the instructions, the experiment proceeded identically to Experiment 2 with the exception that the delay conditions were added.

\section{Results}

To assess accuracy, we calculated difference scores as in Experiment 1 (see Table 1). We calculated a 2 (gender) $\times 4$ (stimulus) $\times 5$ (delay) mixed ANOVA on the difference scores. The grand mean $(M=2.24, S D=2.44)$ was significantly greater than zero, $F(1,110)=255.98, p<.001, M S E=9.4, \eta^{2}=2.32$, indicating that participants estimated the shape of the window as closer to a rectangle (as predicted by shape constancy). There was also a significant effect of stimulus, $F(3,330)=47.2, p<.001$, $M S E=3.46, \eta^{2}=0.42$. Tukey's HSD indicated that, as in Experiment 1, the participants' error when estimating the two extreme views $\left(26^{\circ}\right.$ and $\left.78^{\circ}\right)$ was significantly less than their error when estimating the two less extreme views $\left(52^{\circ}\right.$ and $65^{\circ}$ ) (see Table 1). As with Experiment 1, the error associated with the two extreme views were equivalent and the error associated with the two less extreme views were equivalent. There was no effect of delay $(F<1.0)$ or gender $(F=3.18)$. There were no significant interactions.

\section{Discussion}

The results of Experiment 3 reveal no effect of memory on shape constancy. Specifically, there was no significant difference in the error in judgment between any of the delay conditions. Perhaps more impressively, the delay conditions were not significantly different from the simultaneous viewing condition. Thus, shape constancy is predominantly perceptual in nature and occurs at the time of encoding. This suggests that the shape constancy task measures the artist's ability to encode stimuli accurately.

Together, Experiments 1-3 suggest that the shape constancy task is a robust predictor of drawing accuracy and it measures the ability of the artist to accurately encode the to-be-drawn stimulus. It is unclear, however, whether the shape constancy task taps into the ability to accurately encode featural and/or spatial information. 
Because shape constancy causes errors in holistic perception, it may be that the shape constancy task is more related to spatial perception than featural perception. Nevertheless, those who are skilled at spatial perception may also be skilled at featural perception. In Experiment 4, we explore the relation between perceptual errors resulting from shape constancy and drawing accuracy in more detail by disambiguating featural and spatial drawing accuracy from overall drawing accuracy.

\section{Experiment 4}

In Experiment 4, we further explore the relation between shape constancy and drawing accuracy by assessing the relation between perceptual errors resulting from shape constancy and (1) the ability to accurately render features, and (2) the ability to accurately render spatial relations, (3) the ability to recognize featural details of an image, and (4) the ability to recognize spatial details of an image. Furthermore, in Experiment 4 we assess delayed and simultaneous featural and spatial recognition abilities. These two conditions provide a way to assess the relation between drawing accuracy and the effects of memory and encoding in featural and spatial recognition abilities.

\section{Method}

\section{Participants}

Sixty students volunteered as participants in the drawing, recognition, and constancy tasks. To ensure variability in drawing accuracy, we recruited both novice (had no formal art training) and expert artists (art majors or minors). Thirty-five of the participants were novices who received course credit for their participation and were recruited from the general psychology subject pool. Twentyfive of the participants were experts, who were studio art majors and minors who were recruited from various studio classes, and received extra credit for participating. Participants were naïve with respect to the task.

Four expert critics volunteered to rate the accuracy of the drawings produced by the participants. Two of the critics were art history professors and two of the critics were studio art professors. Expert critics were used because the critics task involved determining the accuracy of featural and spatial aspects of the drawings separately. It was assumed that the experts' familiarity with these concepts would aid in the producing accurate judgments.

\section{Drawing Task Materials}

Eight $6.75 \times 10.25$ in. $(17.5 \times 26.24 \mathrm{~cm})$ gray scale photographs were used in the drawing task. Each photograph depicted the face and shoulders of an adult Caucasian woman who appeared to be about 20 years old. The backgrounds were digitally removed from the images.

Black foam board was used to create a distraction free backdrop on which to mount the photographs. The backdrop was 36.5 in. (wide) by $30 \mathrm{in}$. (tall) and placed $23 \mathrm{in}$. from the front edge of the table. Two hooks were placed on the foam board, which were used to hang the photographs in the center of the board, at or near eye level of the participants. Thus, the photograph was mounted approximately $25 \mathrm{in}$. from the participant and $10 \mathrm{in}$. from the table top.

\section{Recognition Task Materials}

Each photograph was altered to make five images for recognition (termed the recognition images): three featural images, one spatial, and one noisy image (see Figure 5). To create the featural images, the left eye, nose, and mouth were isolated and each embedded in a plain white document. The spatial images were created to remove all featural information, but retain the spatial placement of the features. To accomplish this, for each face, we create gray ovals the size of the face and two small black ovals were positioned where the eyes were located, a vertical rectangle was placed where the nose was located, and a horizontal rectangle was placed where the mouth was located. The noisy images were created to retain both featural and spatial information, but to partially obscure both through noise. To create these images a random $50 \%$ of the pixels were randomly assigned a grayscale value, thus converting the face into a blurred, dot-like image. Each image was placed in a clear plastic page protector. An example of these images can be seen in Figure 5.

The recognition images from the eight photographs were divided into two sets. Each set contained, (1) the five recognition images from the learned face (termed the target recognition images), and (2) the recognition images from three novel faces which served as distractors (termed the distractor recognition images). Each set of 20 images was organized in a three-ring notebook (thus there were two notebooks). The order of the 20 images was randomized within the notebook for each participant. The participants were asked to identify the images from the learned face by responding on a 10-point scale ranging from " 1 : very sure that the image was not from the learned photograph," to "10: very sure the image was from the learned photograph." The scale was centered at the bottom of each image.

\section{Procedure}

Each participant was tested individually. All participants began by completing a drawing task. The drawing procedure was identical to that of Experiment 2, with the exception that participants only drew a single photograph once. Each participant was randomly assigned a face to draw.

After completing the drawing task, the participant completed the shape constancy task. The shape constancy task was identical to Experiment 3 with the exception that only two within subjects delay condition were used: simultaneous and $300 \mathrm{~s}$. We added the 300 -s condition to test whether the results of Experiment 3 were robust for 5-min delays. The two delay conditions were counterbalanced between windows within participant (i.e., responses for two of the four window were delayed by $300 \mathrm{~s}$ and the responses were made in the presence of the remaining two windows). The order that the windows were presented was counterbalanced between participants.

Upon completion of the constancy task, participants were tested on their recognition of featural, spatial, and holistic details of the face that they had drawn in the drawing task (the delay recognition task). For this task, participants were told that they would view a series of pictures that may or may not have been constructed using the photograph that they had previously drawn. Participants were also told that the pictures would include facial features, masks with 


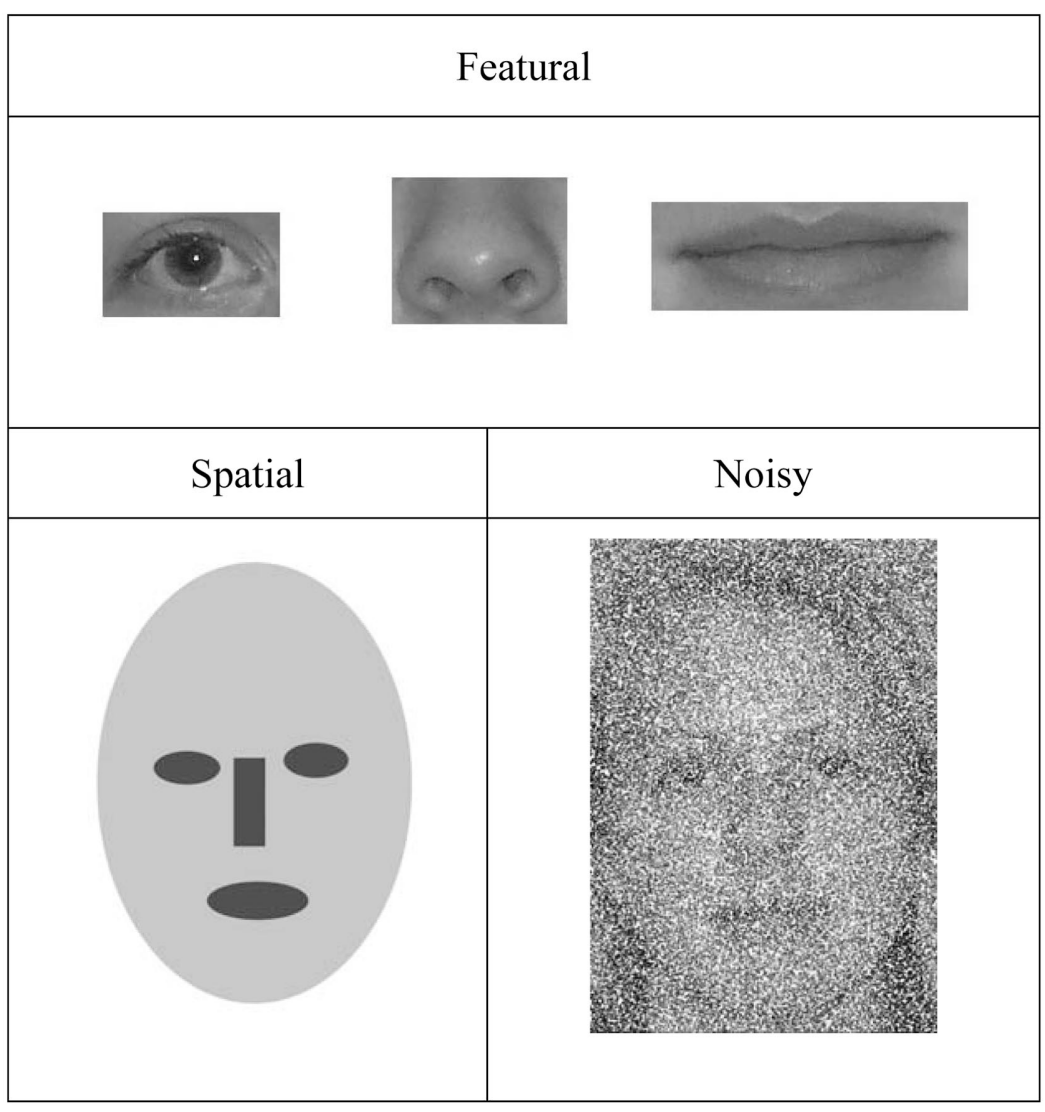

Figure 5. Examples of featural, spatial, and noisy images used in the recognition tasks.

features blacked out, and pictures with added noise. They were instructed to rate their confidence that each picture was from the photograph they drew. The ratings were to be made on a 10-point scale ranging from " 1 : very sure that the image was not from the learned photograph," to "10: very sure the image was from the learned photograph."

The participants were given the facial recognition notebook that corresponded with the photograph they had drawn earlier. The participants rated each image aloud, at their own pace, while the test administrator recorded the scores. After each rating, the page was turned by the participant, and scores could not be changed after the page was turned.

Following the completion of the delay recognition task, the simultaneous recognition task was implemented. The photograph to be recognized (termed the target photograph) in the simultaneous condition was randomly chosen from the four faces not used in the previous recognition task. The recognition images from the three remaining faces were used as distractors. The recognition images were organized in a notebook as described above. The target photograph was mounted in the same manner as the drawn photograph and participants were tested again for recognition. In this condition, the target photograph remained visible during the recognition task. Participants rated each image aloud, on the same 10-point scale used in the delay recognition task, and the experimenter recorded the scores.

\section{Judging}

All the drawings were divided according to the photograph drawn, and then placed into corresponding folders, forming eight folders. Each critic produced three accuracy ratings for each drawing: one based on the featural details, one based on the spatial details, and one for overall accuracy of the drawing. Instructions were given to each judge describing featural details as those based on the parts of the face such as the nose, mouth, and so forth, and spatial details as the relationships between the features of the face. When judging the accuracy of renderings, the critics were presented the relevant images of the face the artists drew to compare to the artists' renderings of the face. That is, when making their featural drawing accuracy ratings, critics were presented the featural images isolated from the photograph of the drawn face to compare with the artists renderings. When making their spatial drawing accuracy ratings, critics were presented the spatial masks derived from the photograph of the drawn face to compare with the artists renderings. When making their overall drawing accuracy ratings, critics were presented the original photograph of the drawn face to compare with the artists renderings.

Critics were asked to rate the accuracy of the drawings on a scale of 1 to 100 , where 1 represented a very poor representation and 100 represented a very accurate representation. All rating documents had a copy of the rating scale on the bottom. 
The critics viewed the entire set of drawings three times: once for each rating. The order was fixed to control for the critics knowledge of the overall composition of each face: (1) featural, (2) spatial, and (3) overall. Each rating was given by the critic at his or her own pace, aloud, and the researcher recorded the response.

\section{Results}

\section{Reliability of Critics Ratings}

To ensure consistent use of the rating scale between critics, we standardized each critic's ratings to have a mean of zero and an $S D$ of one. These standardized ratings were used in all analyses. Cronbach's alpha, $\alpha=.80$, showed that the critics' ratings were remarkably reliable given the subjective nature of the task. The expert critics' Cronbach's alpha is smaller than that of the novices in Experiment 2 primarily because there were only four expert critics versus 50 novice critics in Experiment 2.

The current dataset provides the information necessary to: (1) quantify the relation between perceptual errors resulting from shape constancy and featural and spatial drawing errors, and (2) quantify the relation between perceptual errors resulting from shape constancy and recognition of featural and spatial information. Perceptual errors resulting from shape constancy were calculated using the same method described in Experiment 1.

\section{Mean Effects of Shape Constancy}

To assess the effects of artist skill (novice vs. expert), delay (0 s, $300 \mathrm{~s})$, and window $(6,12,15,18)$ on perceptual errors resulting from shape constancy, we calculated a $2 \times 2 \times 4$ mixed model ANOVA where participant was treated as a random effect. The was a significant main effect of window, $F(3,114)=8.5, p<$ $.001, M S E=5.34, \eta^{2}=0.22$. Tukey's HSD $(p<.05)$ indicated that the participants' error when estimating the two extreme views $\left(26^{\circ}\right.$ and $\left.78^{\circ}\right)$ were equivalent to each other and significantly less than their error when estimating the two less extreme views $\left(52^{\circ}\right.$ and $65^{\circ}$ ), which were also equivalent to one another (see Table 1). This replicates the findings of Experiment 1. There was no effect of delay $(F<1.0)$, thus replicating the lack of influence of memory delay on perceptual errors resulting from shape constancy. There were no other significant main effects or interactions $(p>.05)$. Because of the lack of significant effects, in all remaining analyses the data was collapsed over delay and skill. All remaining analyses were conducted on the perceptual errors resulting from shape constancy averaged over all windows.

\section{Shape Constancy and Drawing Accuracy}

To assess the relation between shape constancy and drawing accuracy, we correlated perceptual errors resulting from shape constancy with featural drawing accuracy, spatial drawing accuracy, and overall drawing accuracy, as judged by the expert critics. There was a significant correlation $(p<.05)$ between perceptual errors resulting from shape constancy and spatial drawing accuracy $(r=-.31)$, and overall drawing accuracy $(r=-.29)$. There was a trend toward a significant relation between perceptual errors resulting from shape constancy and featural drawing accuracy $(r=$ $-.24, p=.067)$.

\section{Shape Constancy and Recognition Accuracy}

For the recognition task, participants rated their confidence that an image was from a specific photograph on a 10-point scale. The recognition ratings were standardized for each participant, such that the score given for each target image (e.g., nose, mask, etc.) was subtracted from the average score of all the ratings for that class of images (e.g., all nose ratings), and divided by the standard deviation of all of that participant's ratings. This provides a measure of relative confidence, where large numbers indicate better detection.

To assess the relation between shape constancy and recognition accuracy, we correlated perceptual errors resulting from shape constancy with recognition for local features, spatial relations, and the noisy image. Recall that participants completed the recognition task for 1) the face they were asked to draw, and 2) a second, new face that was visible during the recognition stage. The former condition occurred after about a 15-min delay and the original face was not visible during the recognition task (termed the delay condition). In the latter condition, the face was visible during the recognition task (termed the simultaneous condition). These two conditions provide a way to disentangle the effects of memory and encoding. Specifically, the simultaneous condition assessed the perceptual skills of the artists without any distortions resulting from memory storage, whereas the delay condition was subject to memory distortion.

It is interesting that shape constancy did not significantly correlate $(p>.05)$ with any recognition task in either the delayed or simultaneous task. There were, however, significant correlations $(p<.05)$ between the recognition of local features in the delay condition and featural drawing accuracy $(r=.40)$, spatial drawing accuracy $(r=.25)$, and overall drawing accuracy $(r=.28)$. Similarly, there were significant correlations $(p<.05)$ between the recognition of local features in the simultaneous condition and featural drawing accuracy $(r=.37)$, spatial drawing accuracy $(r=$ $.33)$, and overall drawing accuracy $(r=.36)$. An interesting finding is that local feature recognition in the delay condition was uncorrelated with local recognition in the simultaneous condition $(r=-.02)$. There were no other significant correlations between the recognition task and drawing accuracy. The finding that perceptual errors resulting from shape constancy, simultaneous feature recognition, and delayed feature recognition all relate to drawing accuracy, but do not relate to one another, indicates that each of the three tasks measure independent skills that are likely contribute to drawing accuracy.

\section{Shape Constancy, Local Recognition, and Drawing Accuracy}

To disentangle the relations of encoding and memory storage with drawing accuracy, we calculated a regression in which overall drawing accuracy was the criterion variable and the predictor variables were: 1) perceptual errors resulting from shape constancy, 2) recognition accuracy of the local features in the delay condition, and 3) recognition accuracy of the local features in the simultaneous condition. The regression was significant $F(3,56)=$ 7.7, $p<.001, r^{2}=.28$. All three predictors were significant contributors to the regression (see Table 2).

To determine the relation between the above predictors and featural and spatial drawing accuracy, we reran the above regression sepa- 
Table 2

Parameter Estimates for Regression Predicting Overall Drawing Accuracy

\begin{tabular}{lcrrc}
\hline & Coefficients & $S E$ & $t$ stat & $P$ value \\
\hline Overall drawing accuracy & & & & \\
$\quad$ Intercept & -1.08 & 0.40 & -2.68 & 0.01 \\
Perceptual errors resulting from shape constancy & -0.12 & 0.05 & -2.24 & 0.03 \\
Feature recognition - delay & 0.40 & 0.18 & 2.25 & 0.03 \\
$\quad$ Feature recognition - simultaneous & 0.51 & 0.16 & 3.23 & 0.002 \\
Featural drawing accuracy & & & & \\
$\quad$ Intercept & -1.42 & 0.38 & -3.69 & $<0.001$ \\
Perceptual errors resulting from shape constancy & -0.08 & 0.05 & -1.69 & 0.10 \\
Feature recognition - delay & 0.61 & 0.17 & 3.60 & $<0.001$ \\
Feature recognition - simultaneous & 0.53 & 0.15 & 3.54 & $<0.001$ \\
Spatial drawing accuracy & & & & \\
Intercept & -0.53 & 0.36 & -1.47 & 0.15 \\
Perceptual errors resulting from shape constancy & -0.11 & 0.05 & -2.41 & 0.02 \\
Feature recognition - delay & 0.24 & 0.16 & 1.51 & 0.14 \\
Feature recognition - simultaneous & 0.33 & 0.14 & 2.37 & 0.02 \\
\hline
\end{tabular}

rately for each of these two drawing accuracy variables. Both the regressions predicting featural drawing accuracy, $F(3,56)=9.9, p<$ $.001, r^{2}=.35$; and spatial drawing accuracy, $F(3,56)=4.5, p=$ $.004, r^{2}=.21$; were significant. However, when predicting featural drawing accuracy, only the two local feature recognition variables were significant, and when predicting spatial recognition, only perceptual errors resulting from shape constancy and local feature recognition in the immediate condition were significant.

\section{Discussion}

The data from Experiment 4 reveal that shape constancy, delayed feature recognition, and simultaneous feature recognition are all unique predictors of overall drawing accuracy. Specifically, 1) perceptual errors resulting from shape constancy and simultaneous feature recognition predict spatial drawing accuracy, and 2) simultaneous and delayed feature recognition predict featural drawing accuracy. These data suggest that spatial drawing accuracy is primarily related to the artist's ability to encode the stimulus accurately (because both predictors of spatial drawing accuracy are tasks that primarily measure encoding skills). In contrast, featural drawing accuracy is related to both feature encoding and storage.

The regression analysis reveals that delayed feature recognition accounts for some unique featural drawing accuracy variance above and beyond that of the feature recognition variable alone. This suggests that delayed feature recognition's capacity to predict featural drawing accuracy is not simply a function of the artist's ability to better encode the stimulus. This finding implicates the memory systems as well as the perceptual systems in predicting drawing accuracy. These results, as well as those of the other three experiments, are discussed in detail below.

\section{General Discussion}

Shape constancy tasks have been used to study artistic ability and vice versa. For example, Mitchell et al. (2005) used a stimulus that induced perceptual errors resulting from shape constancy to study the influence of perception on the drawing process, and in Thouless' (1932) studies of shape constancy, he discovered that professional artists are more resistant to perceptual errors resulting from shape constancy than the average person. In the present paper, we explore the relation between a shape constancy task and drawing accuracy. Experiment 1 validated a simple paper-andpencil shape constancy task. Experiment 2 revealed that this shape constancy task was a robust predictor of drawing accuracy. Experiment 3 revealed that perceptual errors in the shape constancy task were a function of the perceptual encoding process rather than the memory storage process. Finally, Experiment 4 revealed that the shape constancy task specifically predicts spatial drawing accuracy, a delayed feature recognition task predicts feature drawing accuracy, and a feature encoding task predicts both featural and spatial drawing accuracy. These results are best explained within the context of Rock's theory of Constructive Perception (e.g., Rock, 1983).

Rock (1983) makes the distinction between the distal stimulus (i.e., the object in the world) and the proximal stimulus (the pattern of stimulation on our sense organ, in this case, the retina). Rock hypothesizes that the initial perception of a stimulus is fleeting and corresponds relatively closely to the proximal stimulus. This initial perception is followed by an unconscious inference stage whereby the perceptual system identifies the three-dimensional structure of the object. This perceptual solution leads to the final percept, which corresponds more closely to the distal state of affairs than the proximal stimulus. There is abundant evidence to support Rock's hypothesis that cognition influences perception even when the stimulus remains visible (e.g., Epstein, 1977; Rock, 1983, 1996, but see Gibson, 1950).

Integral to Rock's theory of constructive perception is the supposition that, in most cases, both the initial percept and the final percept are potentially available to the observer (Rock, 1983). The initial percept, however, is less relevant to one's ability to accurately identify the shapes and positions of objects in space, and thus is less relevant to survival. As a result, perception is dominated by the final percept.

Assuming that Rock's theory of constructive perception is true, one can only accurately draw a stimulus by rendering the initial percept (i.e., the proximal stimulus). When one draws the proximal stimulus, the observer's perceptual system will apply the same perceptual transformation on the drawn image as is applied to the 
proximal stimulus. This will result in a final percept that appears accurate. The shape constancy task and (perhaps) the feature encoding task likely measure one's ability to access their proximal image and thus predict drawing accuracy.

Drawing errors may result from people attempting to reproduce their final percept rather than their initial percept. It is unclear what would result from drawing the final percept. In essence, because the final percept is (presumably) in three-dimensional space, one cannot reproduce it accurately in a two-dimensional medium without recreating the proximal stimulus that gave rise to the final percept in the first place. It may be this impossibility that leads to the anecdotal confusion that occurs when one is asked to draw a stimulus and the widespread practice of using "drawing rules" to accurately render a stimulus (Gombrich, 1961).

There is evidence that one's final perception of a stimulus has significant impact on one's drawing of that stimulus. In a telling experiment, Van Sommers (1984) demonstrated that participants' interpretations of a to-be-drawn stimulus influenced their stroke directions and positions when drawing the stimulus. Furthermore, Mitchell et al. (2005) demonstrated that the drawing errors in their experiments are driven by the final percepts resulting from perceptual errors resulting from shape constancy.

The cumulative evidence to-date supports the supposition that some people are able to overcome (to some degree) the dominance of the final percept, and these people are better able to accurately draw a stimulus. Cohen (2005) may provide a clue as to how people are able to overcome the dominance of the final percept. Through a series of experiments, Cohen demonstrated that skilled artists' have higher gaze frequencies (i.e., very short glances at the stimulus and the drawing) between the to-be-drawn stimulus and their drawing than those of unskilled artists and that the speed of gaze frequencies is a causal factor in drawing accuracy. High gaze frequencies reduce the influence of the final percept by allowing insufficient time for it to develop. Indeed, there is evidence that perceptual errors resulting from shape constancy are reduced with reduced presentation times (Epstein et al., 1977; Leibowitz \& Bourne, 1956; Leibowitz, Mitchell, \& Angrist, 1954). These findings are consistent with the hypothesis that skilled artists are actively overcoming the influence of the final percept. Specifically, if, as Rock claims, one's initial percept is nearly identical to the proximal stimulus, then high gaze frequencies would create a series of initial percepts.

One unexpected finding of Experiment 4 was that delayed feature recognition predicts featural drawing accuracy over and above the predictive effects of feature encoding. This implies that skilled artists are less susceptible to memory distortion than unskilled artists. Memory distortion has been well-documented in the extant literature. For example, Werner and Diedrichsen (2002) provide evidence that spatial memory begins to distort in remarkably brief time intervals (i.e., $50 \mathrm{~ms}$ ). Furthermore, Huttenlocher, Hedges, and Duncan (1991) show that the direction of memory distortion is influenced by context. It is not inconceivable that memory distortion could play an important role in drawing accuracy. Thus, the role of memory distortion on drawing accuracy should be explored more thoroughly.

The present findings have implications for art education. On a general level, the data and theory presented here provide evidence of the importance of automatic perceptual processes in the creation of art. If this finding holds true, then students would benefit from classes that focus on understanding these perceptual processes and their role in art production. Furthermore, art educators may choose to develop techniques that focus on honing students' perceptual skills, as well as their skill more directly associated with artistic techniques. One can also take these findings a bit beyond the data presented here and consider their potential impact on the field of art history. That is, the limits and processes of the human perceptual system may inform some debates in the field of art history. For example, understanding the limits and processes of the human perceptual system may inform the debate surrounding the role of optics in old masters' art (e.g., Hockney, 2001).

On a more specific level, the data and theory presented here suggest that teaching students to access their retinal image and inhibit their final percept is a crucial step in helping them to learn to draw realistically. There are a variety of techniques currently used in the classroom that aim to promote accurate stimulus perception to facilitate drawing accuracy. These techniques include contour drawing, drawing of negative spaces, and inversion of the stimulus (Edwards, 1986, 1989). These techniques are intended to interfere with the formation of the final percept (in a variety of ways) and thus facilitate the artist's ability to access the retinal image when drawing. For example, inversion of a stimulus is believed to inhibit automatic holistic processing, causing an increased reliance on featural processing. This switch in processing methods is assumed to interfere with the perceptual transformations initiated by object recognition and thus lead to increases in drawing accuracy. However, these techniques have yet to be formally tested, and it is therefore unknown whether their use would lead to an increase in drawing accuracy. It is therefore important for future research to examine the effectiveness of these techniques.

Teachers may also consider creating new exercises to help train students to access their retinal image. Although the current paper does directly not address ways to facilitate access of one's retinal image, Cohen (2005) and Epstein et al., 1977 suggest that quickly alternating one's gaze between one's drawing and the to-be-drawn stimulus will reduce errors associated with perceptual constancy and facilitate accurate drawing. One way in which teachers may help train students to quickly alternate their gazes is to have students perform a task similar to the shape constancy task while instructing them to alternate their gaze at a variety of frequencies. For example, exercises might be developed in which the student views objects from various angles and his or her goal is to identify the correct outline of the object from his or her viewpoint. The teacher may instruct the students to alternate their gaze quickly in one condition and slowly in another and then compare the results. Such exercises may help the student to notice the difference in accuracy under the different conditions. Future experiments should address whether this and other similar exercises facilitate drawing accuracy and reduce shape constancy errors.

In conclusion, there is a robust negative relation between perceptual errors resulting from shape constancy and drawing accuracy. The shape constancy task likely measures one's ability to overcome constructive perception processes that transform the retinal image into a final percept. That is, (1) one's percept of a stimulus is generally different from the projection of the stimulus on the retina; (2) to accurately draw the stimulus, the artist must override, or compensate for, the system that distorts the retinal image; and (3) the shape constancy and the feature encoding tasks 
measure, to some extent, the ability of the observer to override, or compensate for, the system that distorts the retinal image. These present findings support Cohen and Bennett's (1997) hypothesis that the major source of drawing errors lies in the perceptual stage of the drawing process.

\section{References}

Blakemore, C. (1973). The baffled brain. In R. L. Gregory, \& E. H. Gombrich (Eds.). Illusions in nature and art. (p. 9-48). New York: Charles Scribner's Sons.

Blakemore, C., Carpenter, R. H. S., \& Georgeson, M. A. (1970). Lateral inhibition between orientation detectors in the human visual system. Nature, 228, 37-39.

Cohen, D. J. (2005). Look little, look often: The influence of gaze frequency on drawing accuracy. Perception and Psychophysics, 67, 9971009.

Cohen, D. J., \& Bennett, S. (1997). Why can't most people draw what they see? Journal of Experimental Psychology: Human Perception and Performance, 23, 609-621.

Deregowski, J. B. (1973). Illusion and culture. In R. L. Gregory, \& E. H. Gombrich (Eds.). Illusions in nature and art. (p. 161-192). New York: Charles Scribner's Sons.

Edwards, B. (1986). Drawing on the artist within. New York: Simon \& Schuster, Inc.

Edwards, B. (1989). Drawing on the right side of the brain. New York: Putnam.

Epstein, W. (1977). Stability and constancy in visual perception: Mechanisms and processes. New York: Wiley and Sons.

Epstein, W., Hatfield, G., \& Muise, G. (1977). Perceived shape at a slant as a function of processing time and processing load. Journal of Experimental Psychology: Human Perception and Performance, 3, 473-483.

Epstein, W., \& Park, J. N. (1963). Shape constancy: Functional relationships and theoretical formulation. Psychological Bulletin, 60, 265-288.

Freeman, N. H. (1980). Strategies of representation in young children: Analysis of spatial skills and drawing processes. London: Academic Press.

Freeman, N. H. (1987). Current developments in the development of representational picture-production. Archives de Psychologie, 55, 127152.

Gibson, J. J. (1950). The perception of the visual world. Oxford, UK: Houghton Muffin.

Gombrich, E. H. (1961). Art and illusion: A study in the psychology of pictorial representation. Oxford, UK: Pantheon.
Gregory, R. L. (1990). Eye and brain. (4th ed.). Princeton, NJ: Princeton University Press.

Hockney, D. (2001). Secret knowledge: Rediscovering the lost techniques of the old masters. New York: Viking Studio.

Huttenlocher, J., Hedges, L. V., \& Duncan, S. (1991). Categories and particulars: Prototype effects in estimating spatial location. Psychological Review, 93, 352-376.

Kohler, W. (1947). Gestalt psychology. London: Liveright.

Kozbelt, A. (2001). Artists as experts in visual cognition. Visual Cognition, 8, 705-723

Lee, M. (1989). When is an object not an object? The effect of "meaning" upon the copying of line drawings. British Journal of Psychology, 80, 15-37.

Leibowitz, H., \& Bourne, L. E. (1956). Time and intensity as determiners of perceived shape. Journal of Experimental Psychology, 51, 227-281.

Leibowitz, H., Mitchell, E., \& Angrist, N. (1954). Exposure duration in the perception of shape. Science, 120, 400.

Mitchell, P., Ropar, D., \& Ackroyd, K. (2005). How perception impacts drawing. Journal of Experimental Psychology: Human Perception and Performance, 31, 996-1003.

Nunnaly, J. (1978). Psychometric theory. New York: McGraw-Hill.

Reith, E. (1988). The development of use of contour lines in children's drawings of figurative and non-figurative three-dimensional models. Archives de Psychologie, 56, 83-103.

Rock, I. (1983). The logic of perception. Cambridge, MA: MIT Press.

Rock, I. (1996). Indirect perception. Cambridge, MA: MIT Press.

Sze, M. (1956). The way of painting. New York: Vintage Books.

Thouless, R. H. (1931a). Phenomenal regression to the real object. Part I. British Journal of Psychology, 21, 339-359.

Thouless, R. H. (1931b). Phenomenal regression to the real object. Part II. British Journal of Psychology, 22, 1-30.

Thouless, R. H. (1932). Individual differences in phenomenal regression. British Journal of Psychology, 22, 216-241.

Van Sommers, P. (1984). The impact of meaning on executive strategies. Drawing and Cognition, Cambridge, UK: Cambridge.

Werner, S., \& Diedrichsen, J. (2002). The time course of spatial memory. Memory \& Cognition, 30, 718-730.

Willats, J. (1997). Art and representation: New principles in the analysis of pictures. Princeton, NJ: Princeton Press.

Received April 9, 2007

Revision received July 31, 2007

Accepted August 1, 2007 